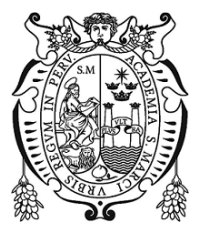

Revista de Investigación de Física 23(3), (2020)

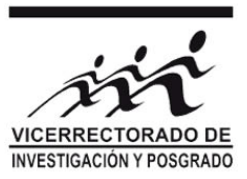

eISSN:1728-2977

\title{
Editorial: Workshop de Mecánica de Fluidos (WMF) "50 Aniversario de la Escuela Profesional de Ingeniería Mecánica de Fluidos" (EPIMF)
}

\author{
Carlos Carbonel ${ }^{*}$ \\ ${ }^{1}$ Universidad Nacional Mayor de San Marcos, Lima, Perú
}

Lima, 01 de diciembre de 2020

En conmemoración del 50 Aniversario de creación de la Escuela Profesional de Ingeniería Mecánica de Fluidos (EPIMF), se desarrolló el Workshop de Mecánica de Fluidos (WMF) del 4 al 6 de Noviembre de 2019 en el Auditorio de la EPIMF, en la Ciudad Universitaria de la Universidad Nacional Mayor de San Marcos (UNMSM), Lima, Perú.

Al evento académico fueron convocados investigadores a presentar artículos asociados a la Mecánica de Fluidos y sus Aplicaciones en los idiomas castellano, portugués e inglés.

El evento contó con el apoyo de la Comisión Científica conformada por los Doctores Julio Aquije Ch., Jorge Bueno G., Carlos Carbonel H., Enrique Huaringa A., Rigoberto Melgarejo M., Franklin Pacheco T., Joel Rojas A., Over Diaz O., Cesar Quispe G.. El desarrollo del evento estuvo a cargo de la Comisión de Ejecución constituida por el Dr. Carlos Carbonel, Dr. Douglas Sarango, Mg. Bernardino Salvador, Ing. Manuel Herquinio A., Mg. Jose Atuncar I.

El presente volumen está basado en los artículos presentados en el WMF, con la aprobación de publicación de sus autores, permitiéndose así, prestigiar el volumen con sus valiosas contribuciones. Parte de los autores y miembros de las comisiones son graduados de la EPIMF en sus 50 años de historia. Es necesario resaltar el esfuerzo por participar de parte de autores provenientes de diversos países congregándose en Lima durante el evento. El contenido del volumen refleja las diversas áreas aplicadas de la Mecánica de Fluidos, la cual es el cordón umbilical de temas y actividades como:
- Manejo de aguas fluviales y aguas subterráneas

- Dinámica marítima costera y de la atmósfera

- Contaminación en medios acuáticos y atmosféricos

- Embalse de relaves, conducción de pulpas

- Flujos térmicos, oleo-hidráulica, neumática

- Aprovechamientos energéticos, térmicos, gas.

- Aprovechamientos hídricos, eólicos, oleajes.

Históricamente la visión de Ingeniería Mecánica de Fluidos fue promovida por el Dr. Rafael Davila Cuevas en estrecha colaboración con la misión francesa dirigida en aquel entonces por el ing. Bernard Guy Gerlier, estructurándose el Plan de Estudios inicial en 1967-1968. Se fundo en la Facultad de Ciencias en 1969, época en la cual se realizó el cambio del Sistema Facultativo al Departamentalísta en la UNMSM. El "Programa de Ingeniería de Mecánica de Fluidos", fue incluido en el Departamento de las Ingenierías bajo la dirección del Dr. Ricardo Lama Ramirez, como "Escuela Académico Profesional de Ingeniería Mecánica de Fluidos".

Uno de los avances estructurales académicos mas significativos, asociado con la EPIMF, se alcanzó en el año 2005. Se inicio el Maestrado en Hidráulica Computacional, un área de avanzada en la Ingeniería. En esa ocasión, se contó con el apoyo de una nueva Misión Francesa del ENSEEIHT (Tolouse, Francia) liderada por el Dr. Dominique Legendre.

Actualmente la EPIMF cuenta con un Pabellón y Laboratorios para el desarrollo de calidad de la formación ingenieril requerida. Los logros alcanzados en los primeros 50 años muestran que el futuro de la Ingeniería Mecánica de Fluidos, todavía se esta construyendo!!

*ccarbonelh@unmsm.edu.pe 